\title{
Impact of Laser Guide Star Fratricide on TMT MCAO System
}

\author{
Lianqi Wang, ${ }^{a}$ Angel Otarola ${ }^{a}$, Brent Ellerbroek ${ }^{a}$ \\ aThirty Meter Telescope Project, 2632 E Washington Blvd, Pasadena, CA, 91107, USA
}

\begin{abstract}
Laser beams projected from the ground to form laser guide stars (LGS) experience scattering and absorption that reduce their intensity as they propagate through the atmosphere. Some fraction of the scattered light will be collected by the other LGS wavefront sensors and causes additional background in parts of the pupil. This cross-talk is referred to as the fratricide effect. In this paper we quantify the magnitude of four different sources of scattering/absorption and back scattering, and evaluate their impact on performance with various zenith angles and turbulence profiles for the Thirty Meter Telescope (TMT) MCAO system, NFIRAOS. The resulting wavefront error is on the order of 5 to $20 \mathrm{~nm}$ RMS, provided that the mean background from the fratricide can be calibrated and subtracted with an accuracy of $80 \%$. We have also found that the impact of fratricide is a weak function of LGS asterism radius.
\end{abstract}

\section{INTRODUCTION}

Current $^{1-3}$ and next generation ${ }^{4,5}$ ground based astronomical telescopes rely upon laser guide star adaptive optics systems to mitigate the image blurring due to atmospheric turbulence to achieve diffraction limited resolution. Sodium laser guide star (LGS) multi-conjugate adaptive optics (MCAO) is a popular concept to mitigate the image blurring in a modest wide field of view (tens of arcseconds) by measuring the turbulence along several different directions and reconstructing a 3-D profile of the turbulence.

When the laser beam passes through the atmosphere, it will experience scattering and absorption that reduce its intensity. Some fraction of the light lost due to scattering will be back scattered in the direction of the transmitter. In a LGS MCAO system such as the thirty meter telescope (TMT) narrow field infrared adaptive optics system (NFIRAOS), a fraction of the back scattered light from each of the laser beams ends up being collected by WFSs observing the other LGSs, which is generally referred to as the fratricide effect. The fratricide effect is mostly prominent for MCAO systems that launches all the laser beams from near the center of the primary mirror (e.g., behind the secondary mirror) and is stronger at higher zenith angles.

The important sources of scattering or absorption in the atmosphere are Rayleigh scattering, ozone Chappius band absorption, aerosol, and cirrus clouds scattering. The scattered (or re-emitted) light is usually not isotropic. The so called phase function describes the angular distribution of the scattered light. When the scattering particle is much smaller than the wavelength of the incident photon (i.e., in the regime of Rayleigh scattering), the phase function is symmetric toward forward and backward directions. On the other hand, when the scattering particle is similar to or larger than the wavelength of the incident photon (i.e., in the regime of Mie scattering), the forward scattering is dominant. The aerosol and cirrus clouds scattering fall to this latter category and therefore does not contribute significantly to the fratricide effect.

In this paper, we describe the methods to quantify the sources of scattering and/or absorption, as well as the back scattered light that caused the fratricide effect. We will present the results on the impact of fratricide on the performance of TMT NFIRAOS system at different zenith angles and seeing conditions. We have found that the measured Mauna Kea 13N (MK13N) 25th and 50th percentile seeing profile, with $80 \%$ level calibration of the fratricide effect the incremental wavefront error is less than $10 \mathrm{~nm}$ RMS for zenith angles up to 45 degrees. We also present the results on the impact on system performance due to momentary reduction of LGS signal levels due to increased absorption. A $20 \%$ reduction in the laser return signal level as collected by the wavefront sensors gives a greater impact on the system performance than the fratricide effect for most cases.

Further author information: (Send correspondence to Lianqi Wang) Lianqi Wang: lianqiw@tmt.org

Adaptive Optics Systems II, edited by Brent L. Ellerbroek, Michael Hart, Norbert Hubin, Peter L. Wizinowich, Proc. of SPIE Vol. 7736, 77360G - @ 2010 SPIE - CCC code: 0277-786X/10/\$18 · doi: 10.1117/12.857348 
The remainder of the report is organized as follows. Section 2 describes the sources of the absorption/scattering and presents the calculation of their volume scattering coefficient and optical depth. Section 3 describes the modeling of the wavefront sensor images due to the fratricide effect. Section 4 presents the results.

\section{SOURCES OF FRATRICIDE AND THEIR OPTICAL DEPTH}

In order to quantify the fratricide effect, we first need to compute the volume scattering/absorption coefficients and corresponding optical depth for the various atmospheric constituents that are relevant for this study.

\subsection{Rayleigh scattering}

The total Rayleigh-scattering cross section per molecule, $\sigma$, is given by the following formula ${ }^{6-9}$

$$
\sigma(\lambda)=\frac{24 \pi^{3}\left(n_{s}^{2}-1\right)^{2}}{\lambda^{4} N_{s}^{2}\left(n_{s}^{2}+2\right)^{2}}\left(\frac{6+3 \rho_{n}}{6-7 \rho_{n}}\right)
$$

where $N_{s}$ and $n_{s}$ are the molecular number density and the refractive index of standard air. The standard air is defined as dry air containing $0.03 \% \mathrm{CO}_{2}$ by volume, at normal pressure $760 \mathrm{~mm} \mathrm{Hg}$ or $101325 \mathrm{~Pa}$, and a temperature of $15^{\circ} \mathrm{C}$. According to Lorenz-Lorentz and Clausius-Mossotti theory, the quantity $\left(n_{s}^{2}-1\right) /\left(n_{s}^{2}+2\right)$ is proportional to the molecular number density $N_{s}$, therefore $\sigma(\lambda)$ is actually independent of both $n_{s}$ and $N_{s}$, which makes sense. The last term,

$$
F_{k}=\frac{6+3 \rho_{n}}{6-7 \rho_{n}},
$$

is the King correction factor, computed from the depolarization factor $\rho_{n}$, which is a function of $\lambda$. This term accounts for the anisotropy of air molecules.

The amount of scattering for a volume of gas in the atmosphere is characterized by the total Rayleigh volume-scattering coefficient $\beta$,

$$
\beta(\lambda, h)=N(h) \sigma(\lambda)
$$

where $N(h)$ is the number density as a function of altitude $h$. The light loss during radiation transfer is

$$
\frac{\mathrm{d} I}{I}=-\beta(\lambda, h) \mathrm{d} h
$$

which gives

$$
I(h)=I\left(h_{0}\right) \exp \left[-\int_{h_{0}}^{h} \beta(\lambda, h) \mathrm{d} h\right] .
$$

The value of integral is conveniently called the optical depth $\tau$. For the laser beacon launched from the observatory, we have

$$
\tau\left(\lambda, h_{0}\right)=\int_{h_{t}}^{h_{0}} \beta(\lambda, h) \mathrm{d} h,
$$

where $h_{t}$ is the altitude of the transmitter.

The angular distribution of the scattered light is described by the Rayleigh phase function $P_{\text {ray }}$. For unpolarized incident radiation, $P_{\text {ray }}$ is simply

$$
P_{\text {ray }}(\theta)=\frac{3}{4}\left(1+\cos ^{2} \theta\right)
$$

However, because molecular anisotropy also affects the angular distribution of Rayleigh-scattered light, a more accurate formula is given by:

$$
P_{\text {ray }}(\theta)=\frac{3}{4(1+2 \gamma)}\left[(1+3 \gamma)+(1-\gamma) \cos ^{2} \theta\right]
$$




\begin{tabular}{cccccc}
\hline $\begin{array}{c}\text { Layer } \\
i\end{array}$ & $\begin{array}{c}\text { Base altitude } \\
h_{i}(\mathrm{Km})\end{array}$ & $\begin{array}{c}\text { Standard } \\
\text { temperature } \\
(\mathrm{K})\end{array}$ & $\begin{array}{c}\text { Temperature } \\
\text { lapse rate } \Gamma_{i} \\
(\mathrm{~K} / \mathrm{km})\end{array}$ & $\begin{array}{c}\text { Static Pressure } \\
P_{i}(\mathrm{~Pa})\end{array}$ & $\begin{array}{c}\text { Back scatter } \\
\text { coefficient scaling } \\
\frac{P_{i}}{P_{s}} T_{s}\end{array}$ \\
\hline 0 & 0 & 288.15 & -6.5 & 101325 & 1.0000 \\
1 & 11 & 216.65 & 0.0 & 22632.1 & 0.2971 \\
2 & 20 & 216.65 & 1.0 & 5474.89 & 0.0719 \\
3 & 32 & 228.65 & 2.8 & 868.019 & 0.0108 \\
4 & 47 & 270.65 & 0.0 & 110.906 & 0.0012 \\
5 & 51 & 270.65 & 2.8 & 66.9389 & 0.0007 \\
6 & 71 & 214.65 & -2.0 & 3.95642 & 0.0001 \\
\hline
\end{tabular}

Table 1: The US standard air model

with

$$
\gamma=\frac{\rho_{n}}{2-\rho_{n}} .
$$

The angular volume-scattering coefficient $\beta(\lambda, h, \theta)$ is then

$$
\beta(\lambda, h, \theta)=\beta(\lambda, h) \frac{P_{\text {ray }}(\theta, \lambda)}{4 \pi} .
$$

For the sodium laser wavelength at $589 \mathrm{~nm}, \gamma=1.395 \times 10^{-2}$, therefore

$$
P_{\text {ray }}(\theta)=0.73 \times\left(1.0418+0.986 \cos ^{2} \theta\right) .
$$

\subsubsection{Standard air at $15{ }^{\circ} \mathrm{C}$}

For the standard air, we have

$$
\beta_{s}(\lambda)=N_{s} \sigma(\lambda)
$$

the value of $\sigma(\lambda)$ is adopted from: ${ }^{9}$

$$
\sigma(\lambda=589 \mathrm{~nm})=3.304 \times 10^{-27} \mathrm{~cm}^{2},
$$

with $\lambda$ expressed in $\mu m$. The molecular number density for standard air is

$$
N_{s}=2.54743 \times 10^{19} \mathrm{~cm}^{-3},
$$

therefore

$$
\beta_{s}(\lambda=589 \mathrm{~nm})=8.406519 \times 10^{-3} \mathrm{~km}^{-1} .
$$

\subsubsection{Real air using the US standard atmosphere model}

The US standard atmosphere model ${ }^{10}$ treats the air as dry, ideal gas. The Rayleigh volume scattering coefficient for the standard atmosphere model is related to the standard $\beta_{s}$ as

$$
\beta(\lambda, h)=\beta_{s}(\lambda) \frac{N(h)}{N_{s}}=\beta_{s}(\lambda) \frac{P(h)}{P_{s}} \frac{T_{s}}{T(h)},
$$

where $P_{s}$ and $T_{s}$ represent the reference pressure and temperature, $P_{s}=101325 \mathrm{~Pa}$ and $T_{s}=288.15 \mathrm{~K}$. The US standard air model describes the annually averaged atmospheric temperature using 7 layers. For each layer, the temperature change approximately linearly with altitude with a lapse rate. Table 1 listed the temperature and pressure properties of each layer. We use this standard air model to approximate the air above Mauna Kea. The error in the computed optical depth caused by this approximation is within $1 \%$ when compared to results by others and can be ignored for our purposes. 

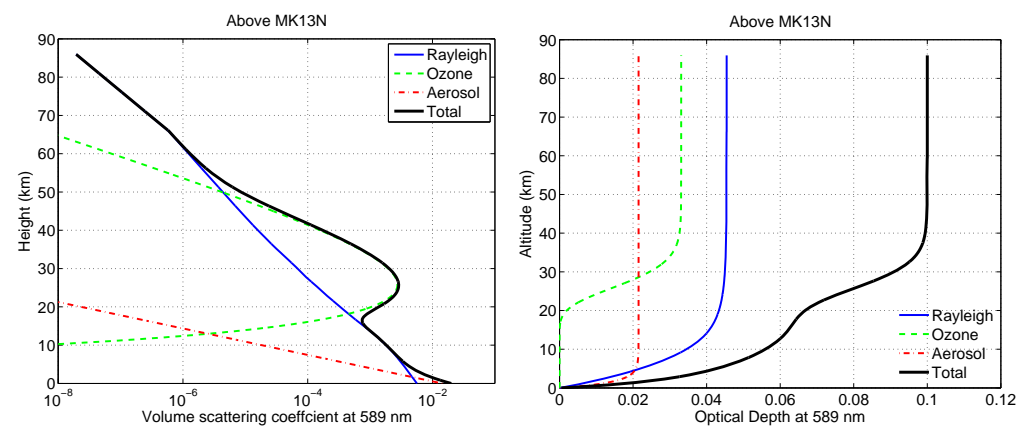

Figure 1: Volume scattering coefficient and optical depth for Rayleigh, ozone Chappius band, and aerosol scattering computed above the TMT MK13N site.

Let $h_{i}$ be the base altitude of layer $i$, the temperature in that layer is given by

$$
T=T_{i}+\Gamma_{i}\left(h-h_{i}\right),
$$

where $T_{i}$ is the standard temperature (base) at the bottom of that layer. From the ideal gas law, $P=\rho R T$. From the hydrostatic equilibrium we have

$$
\frac{\mathrm{d} P}{\mathrm{~d} h}=-g \rho=-g \frac{P}{R T}=-\frac{g}{R} \frac{P}{T_{i}+\Gamma_{i}\left(h-h_{i}\right)},
$$

or

$$
\frac{\mathrm{d} P}{P}=-\frac{g}{R} \frac{\mathrm{d} h}{T_{i}+\Gamma_{i}\left(h-h_{i}\right)}
$$

Therefore,

$$
\frac{P}{P_{i}}= \begin{cases}\left(1+\frac{\Gamma_{i}\left(h-h_{i}\right)}{T_{i}}\right)^{-\frac{g}{R \Gamma_{i}}}=\left(\frac{T}{T_{i}}\right)^{-\frac{g}{R \Gamma_{i}}} & \Gamma_{i} \neq 0 \\ \exp \left[-\frac{g\left(h-h_{i}\right)}{R T_{i}}\right] & \Gamma_{i}=0 .\end{cases}
$$

The constants used here are $g=9.80665 \mathrm{~ms}^{-2}, R=8.31432 \mathrm{~J} /($ mole $\cdot \mathrm{K}) / 0.0289644 \mathrm{~kg} / \mathrm{mole}=287.053 \mathrm{~J} /(\mathrm{kg} \cdot \mathrm{K})$. The change of $g$ is neglected since the altitude is small compared to the radius of the Earth.

Based on the above information, we are ready to compute the Rayleigh volume scattering coefficient and the optical depth at any altitude. Figure 1 plots the Rayleigh volume scattering coefficients and up-link optical depth as a function of the distance above the TMT MK13N site at an altitude of $4050 \mathrm{~m}$. The total Rayleigh optical depth from MK13N up to free space is about 0.0443 .

A direct fit to the Rayleigh volume scattering coefficients for the standard atmosphere model at $0.589 \mu \mathrm{m}$ above the TMT MK13N site gives

$$
\beta(h)=0.00599 e^{-\frac{h}{6.9491}}
$$

where $\mathrm{z}$ is expressed in $\mathrm{km}$. The scale height is $6.95 \mathrm{~km}$. The precise values are used during the computation, instead of this fit.

\subsection{Chappius band of ozone absorption}

The Chappius band of ozone is the wavelength band of light absorbed by ozone in the visible region, approximately 380 to $750 \mathrm{~nm}$. (The ultraviolet absorption region of ozone is called by Hartley-Huggins band.)

The vertical distribution of the numerical density of ozone ${ }^{11}$ can be approximated by

$$
N_{O_{3}}(h)=5.7 \times 10^{18} \exp \left[-((\log (h)-3.25) / 0.26)^{2}\right] \mathrm{m}^{-3} .
$$

with $h$ in $\mathrm{km}$. The scattering cross section ${ }^{12}$ is

$$
\sigma_{O_{3}}=4.8 \times 10^{-25} \mathrm{~m}^{2} .
$$




\begin{tabular}{cc}
\hline Source & Optical Depth \\
\hline Rayleigh & 0.0443 \\
Ozone & 0.0331 \\
Aerosol & 0.0226 \\
Cirrus & 0.2200 \\
\hline
\end{tabular}

Table 2: Total optical depth contributions from Rayleigh, ozone Chappius band, aerosol scattering, and cirrus clouds.

The volume scattering coefficient and optical depth can be calculated in the same way of the Rayleigh back scattering. Figure 1 shows the result. The total optical depth for ozone Chappius band absorption above MK13N is about 0.0331 .

The stratospheric ozone does not play an important role in the overall molecular back scattering because the stratosphere is in a stat of local thermodynamic equilibrium, which implies that the time between molecular collisions is shorter than that of spontaneous emission of radiation. Therefore we just set the phase function to zero.

\subsection{Aerosol scattering}

Atmospheric aerosols are very diverse. They include tiny grains of mineral dust stirred up from the ground, particles of salt left when droplets of sea spray evaporate, bacteria, pollen grains, mold spores, other biosol particles, etc. Most of these are produced at or near ground level, and are washed out of the atmosphere by condensation of cloud droplets on them, followed by precipitation. The aerosols all tend to be concentrated in the lowest part of the atmosphere. An exponential distribution with scale height of about $1.5 \mathrm{~km}$ is a rough approximation to their vertical distribution. ${ }^{13}$

At the gemini website, ${ }^{14}$ the overall extinction for photometric air is roughly $m=0.11 \mathrm{mag} /$ airmass at 589 $\mathrm{nm}$ for Mauna Kea. The conversion between extinction expressed in magnitude $m$ and optical depth $\tau$ can be computed by

$$
e^{-\tau}=10^{-\frac{m}{2.5}},
$$

or

$$
\tau=0.9210 \mathrm{~m} .
$$

Therefore, the optical depth at Mauna Kea is about 0.10. Subtracting the optical depth of Rayleigh scattering and ozone Chappius band absorption, the optical depth for Aerosol is about 0.0226, which is in agreement with the measurements in. ${ }^{15}$

The phase function of aerosol scattering is very different from Rayleigh scattering, since the aerosol particle sizes that have strong scattering are generally assumed to be close to the wavelength of scattered light (This assumption will be verified when data becomes available). The wavelength dependence of aerosol scattering is almost inversely proportional to the wavelength $\left(\alpha \lambda^{-1.2}\right.$ power law is often used). The fraction of back scattered light $\frac{P_{\text {Aerosol }}(\pi)}{4 \pi}$ in units of per solid angle is $0.0035 \mathrm{str}^{-1}$, roughly 40 times smaller than $0.12 \mathrm{str}^{-1}$ of the Rayleigh scatter.

\subsection{Cirrus clouds}

Cirrus are ice clouds that form at temperature below -40C. ${ }^{16}$ Near the tropics, like Mauna Kea, cirrus happens above $8 \mathrm{~km}$, with a mean altitude of $13.5 \mathrm{~km}$ above sea level. The mean thickness is $1.6 \mathrm{~km}$. The vertical distribution can be approximated by a Gaussian. The average value for the total optical depth is about 0.22 . For a reference, the cirrus cloud frequency over the Hawaii region in the period Oct/November 2003, obtained from the ICESat/GLASS and MODIS/Terra missions is in the order of $25 \%$ [See Figures 3(a) and 3(b) in ${ }^{16}$ ]. The size of cirrus ice particles are larger than or similar to the wavelength of visible light, so the fraction of back scatter is similar to aerosol scatter.

\subsection{Summary}

Figure 1 plots the volume scattering coefficients and up-link optical depth of the four effects excluding cirrus as a function of the distance above the TMT Mk13N site at an altitude of $4050 \mathrm{~m}$. Table 2 summarizes the contributions of these 4 sources to optical depth. Temporal variations in cirrus and aerosols will impact atmospheric transmittance much more than back scatter, for typical particle size distributions. 


\section{FRATRICIDE MODELING}

Here we are going to ignore the diffraction effects, and model the fratricide using geometric optics. The light intensity in the sky on a horizontal plane at range $h$ above the telescope is

$$
\begin{aligned}
I(\mathbf{x}, h) & =e^{-\tau(h)} \sum_{i=1}^{N} I_{i}(\mathbf{x}, h)=e^{-\tau(h)} \sum_{i=1}^{N} \rho_{i} L_{i}(\mathbf{x}), \\
L_{i}(\mathbf{x}) & =\operatorname{circ}\left[\frac{\mathbf{x}-\left(\mathbf{r}_{i}+h \boldsymbol{\theta}_{i}\right)}{R_{L L T}}\right] \exp \left[-\frac{\left(\mathbf{x}-\left(\mathbf{r}_{i}+h \boldsymbol{\theta}_{i}\right)\right)^{2}}{2 \sigma^{2}}\right],
\end{aligned}
$$

where $\mathbf{x}$ is the coordinate on the horizontal plane, $\mathbf{x}_{i}, \theta_{i}$ are the location and beam direction of the laser launch telescope for WFS $i$, and therefore $\mathbf{x}_{i}+h \theta_{i}$ is the center of the beam on the plane at range $h . N$ is the number of LGS WFS ( $N=6$ for NFIRAOS), $L_{i}(\mathbf{x})$ is the beam intensity function for the Laser launch telescope for WFS $i$ as further described in Section 7.1, and finally $\rho$ is the on axis intensity of the laser beam at the ground level in units of power per unit area in units of $\mathrm{Wm}^{-2}$, computed from the laser power and the total transmittance of the uplink LGS facility optics. The intensity $I(\mathbf{r}, h)$ also has units of power per unit area. The function $\tau(h)$ is one-way up-link sky optical depth, which is related to the volume scattering coefficient $\beta(h)$ by

$$
\tau(h)=\int_{h_{t}}^{h} \mathrm{~d} h^{\prime} \beta\left(h^{\prime}\right) .
$$

The scattered light intensity back towards the telescope is

$$
S(\mathbf{x}, h)=e^{-\tau(h)} \beta(h, \pi) I(\mathbf{x}, h),
$$

where $\beta(h, \pi)=\beta(h) \frac{P(\pi)}{4 \pi}$ is volume back scattering coefficient as described in Section 2 and the additional factor of $e^{-\tau(h)}$ is due to the light loss when the scattered light propagates back downward. The scattered light intensity $S(\mathbf{x}, \mathrm{h})$ has units of power per unit volume per unit solid angle, $\mathrm{Wm}^{-3} \mathrm{sr}^{-1}$.

The integrated intensity of the scattered light on LGS WFS $j$, subaperture $k$, pixel $l$ is then

$$
P_{j k l}=\gamma_{t} \int \mathrm{d} \phi d_{j k l}(\boldsymbol{\phi}) \int \mathrm{d} \mathbf{x} S_{j k}(\mathbf{x}) \int \mathrm{d} h S(\mathbf{x}+h \boldsymbol{\phi}, h),
$$

where $\gamma_{t}$ is the total optical throughput of the telescope and the LGS path in the instrument, $d_{j k l}(\phi)$ is the detector pixel influence function (the field of view function of the pixel), $S_{j k}(\mathbf{x})$ is the subaperture pupil function, and $\mathbf{x}$ is the pupil plane coordinate. Rewrite this equation as

$$
\begin{aligned}
P_{j k l} & =\gamma_{t} \int \mathrm{d} \phi d_{j k}\left(\boldsymbol{\phi}-\boldsymbol{\phi}_{j k l}\right) \int \mathrm{d} \mathbf{x} S_{0}\left(\mathbf{x}-\mathbf{x}_{k}\right) \int \mathrm{d} h e^{-2 \tau(h)} \beta(h, \pi) \sum_{i=1}^{N} \rho_{i} L_{i}(\mathbf{x}+h \boldsymbol{\phi}) \\
& =\gamma_{t} \sum_{i=1}^{N} \rho_{i} \int \mathrm{d} h e^{-2 \tau(h)} \beta(h, \pi) \int \mathrm{d} \boldsymbol{\phi} d_{j k}(\boldsymbol{\phi}) \int \mathrm{d} \mathbf{x} S_{0}(\mathbf{x}) L_{i}\left(\mathbf{x}+\mathbf{x}_{k}+h \boldsymbol{\phi}+h \boldsymbol{\phi}_{j k l}\right), \\
\boldsymbol{\phi}_{j k l} & =\frac{\mathbf{r}_{j}-\mathbf{x}_{k}}{H}+\boldsymbol{\phi}_{l},
\end{aligned}
$$

where $\phi_{j k l}$ is the center of the FoV for pixel $l$ in subaperture $k$ for WFS $j, \mathbf{r}_{j}$ is the location of the guide star for WFS $j$ on sky, $H$ is the height of guide star, and $\phi_{l}$ is the angular offset of pixel $l$ in its subaperture (expressed in the global x-y coordinate system). The transmittance $e^{-2 \tau(h)}$ comes from the up- and down-ward light loss. The pixel function $d_{j k}$ defines the boundary of the pixels in subaperture $k$ of WFS $j$ for the polar coordinate CCD . $S_{0}(\mathbf{x})$ is the rectangular function defining the subaperture.

Let's treat the final line of 31 in detail. Define $P_{j k l}(h)$ as

$$
P_{j k l}(h)=\gamma_{t} \int \mathrm{d} \phi d_{j k}(\phi) \int \mathrm{d} \mathbf{x} S_{0}(\mathbf{x}) L_{i}\left(\mathbf{x}+\mathbf{x}_{k}+h \phi+h \phi_{j k l}\right) .
$$


This equation contains two convolutions over $\phi$ and $\mathbf{x}$. Rewrite the integral as

$$
\begin{aligned}
P_{j k l}(h) & =\gamma_{t} \int \mathrm{d} \boldsymbol{\phi} d_{j k}(\boldsymbol{\phi}) \int \mathrm{d} \boldsymbol{\kappa} \hat{S}_{0}(\boldsymbol{\kappa}) \int \mathrm{d} \mathbf{x} e^{2 \pi i \boldsymbol{\kappa} \cdot \mathbf{x}} L_{i}\left(\mathbf{x}+h \boldsymbol{\phi}+\mathbf{x}_{k}+h \boldsymbol{\phi}_{j k l}\right) \\
& =\gamma_{t} \int \mathrm{d} \boldsymbol{\kappa} \hat{S}_{0}(\boldsymbol{\kappa}) \hat{L}_{i}(-\boldsymbol{\kappa}) \int \mathrm{d} \boldsymbol{\phi} d_{j k}(\boldsymbol{\phi}) e^{-2 \pi i \boldsymbol{\kappa}(h \boldsymbol{\phi})} \exp \left[-2 \pi i \boldsymbol{\kappa} \cdot\left(\mathbf{x}_{k}+h \boldsymbol{\phi}_{j k l}\right)\right] \\
& =\gamma_{t} \mathcal{F}^{-1}\left[\hat{S}_{0}(\boldsymbol{\kappa}) \hat{L}_{i}(-\boldsymbol{\kappa}) \hat{d}_{j k}(h \boldsymbol{\kappa})\right]\left(-\mathbf{x}_{k}-h \boldsymbol{\phi}_{j k l}\right) .
\end{aligned}
$$

This is the inverse Fourier transform of the product of the Fourier transforms of (i) the subaperture function $S_{0}$, (ii) LLT beam amplitude function $L_{i}$, and (iii) the detector pixel influence function $d_{j k}$, evaluated at locations determined by the subaperture position and the pixel line of sight.

In the appendix, we describe the computation of the Fourier transform of the LLT pupil amplitude function $L_{i}$, subaperture function $S_{0}$, and the detector pixel influence function $d_{j k}$. Substituting those results, (assuming an un-truncated Gaussian laser beam) we get

$$
\begin{aligned}
P_{j k l}(h)= & \gamma_{t} \mathcal{F}^{-1}\left\{2 \pi \sigma^{2} \exp \left[-2 \sigma^{2} \pi^{2}|\boldsymbol{\kappa}|^{2}\right] d^{2} \operatorname{sinc}\left(k_{x} d\right) \sin \left(k_{y} d\right)\right. \\
& \cdot \Delta^{2} \operatorname{sinc}\left[h\left(k_{x} \cos \theta+k_{y} \sin \theta\right) \Delta\right] \operatorname{sinc}\left[h\left(k_{x} \sin \theta-k_{y} \cos \theta\right) \Delta\right] \\
& \left.\cdot \exp \left[-2 \pi^{2} \phi_{b l u r}^{2} h^{2}\left(k_{x}^{2}+k_{y}^{2}\right)\right]\right\}\left(\mathbf{r}_{i}+h \boldsymbol{\theta}_{i}-\mathbf{x}_{k}-h \boldsymbol{\phi}_{j k l}\right) .
\end{aligned}
$$

We can see that $P_{j k l}(h)$ is only dependent on the separation between the center of the laser beam $\mathbf{r}_{i}+h \boldsymbol{\theta}_{i}$ and the mapped detector pixel location $\mathbf{x}_{k}+h \phi_{j k l}$ at range $h$, which is expected.

\section{TIME CONSTANT BACK SCATTER VALUES}

\subsection{Parameter summary}

We are assuming constant back scatter patterns that are independent of time. This assumption is valid as long as the variations have a time scale of minutes or more because the LGS pixel processing algorithm parameters are updated at a rate of about $0.1 \mathrm{~Hz}$. We are using the NFIRAOS asterism geometry, which is an order $60 \times 60$ dual conjugate AO system with 6 LGS WFS arranged in a Pentagon with 35" radius plus one more on-axis. Each of the 6 LGS has a laser power of $25 \mathrm{~W}$. They are all launched from a single LLT of diameter $0.4 \mathrm{~m}$ behind the TMT secondary mirror. The total transmittance of the uplink LGS facility is 0.75 . Each LGS WFS is employing a radial format CCD, where pixels in each subaperture are aligned along the direction of elongation to minimize the required number of pixels. The total transmittance of the telescope and the LGS path in the instrument is $0.81 \times 0.54=0.44$. The column density of the sodium atoms is assumed to be $3 \times 10^{13}$ ions $/ \mathrm{m}^{2}$. The unsaturated coupling efficiency between the scattered photon counts per second per steradians of a single atom and the laser power density is 144 photons $/ \mathrm{s} /\left(\mathrm{W} / \mathrm{m}^{2}\right) /$ ion $/ \mathrm{sr}$. The equivalent transmittance due to imperfect laser beam quality is assumed to be 0.8 . Based on these parameters, we obtain a total of 900 photon detection events per subaperture per frame at $800 \mathrm{~Hz}$ at zenith for sodium laser guide star return. The coupling efficiency value of 144 is slightly larger than the TMT requirement of 130, because we maintained the signal level as the TMT requirement but are using a smaller atmospheric transmittance of 0.78 instead of 0.84 than in the TMT requirement. Constrained matched filter ${ }^{17}$ is used to precess the subaperture images to get wavefront gradient measurements. The constrained matched filter has better performance than centroid algorithms when noise or bright background is present, especially on pixels where the weighting used by the matched filter is low.

Figure 2 shows the total back scatter images (excluding sodium layer return) with telescope at zenith. The sub-image at the location of each subaperture is the subaperture image of size $16 \times 6$ along radial/azimuthal direction (The number of pixels in each subaperture varies from $6 \times 6$ to $15 \times 6$ in the real detector, but in simulations we are assuming that all subapertures have $16 \times 6$ pixels). Each pixel extends 0.5 " on sky. Only WFS 1 (on axis) and 2 (off axis) are shown. The other WFS will be similar to the image of WFS 2 with an rotation according to the geometric orientation. The red circle depicts the LGS location for the observing WFS and the green circles depict other LGS that produce the back scatter. The values in the color bar are the color coding of the intensity of individual pixel in the subapertures. The Rayleigh back scatter is the dominant term as the aerosol and cirrus scattering are highly concentrated in the inner subapertures because of low altitude origin and weak back scatter phase function. 

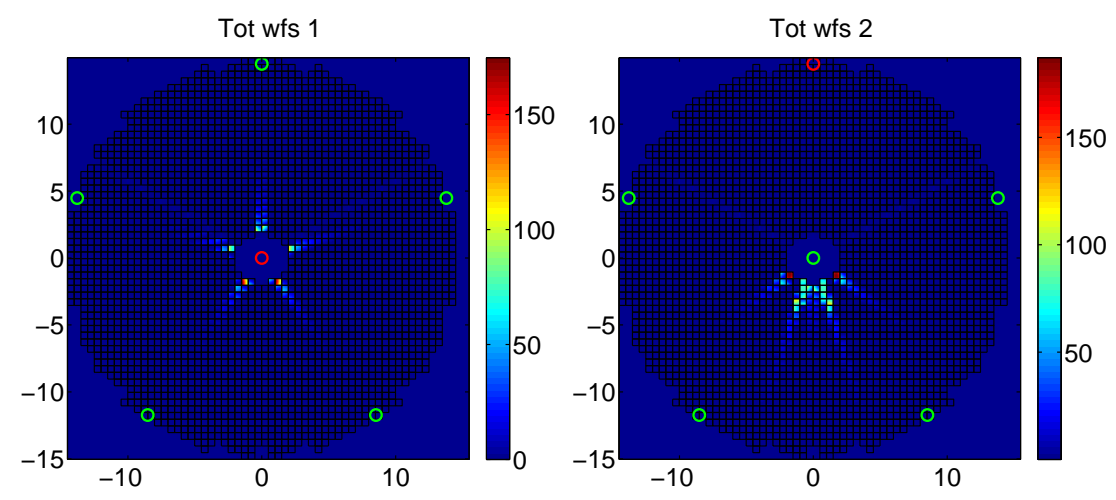

Figure 2: Total back scatter image and histogram of signal levels

\section{IMPACT ON NFIRAOS PERFORMANCE}

The impact of the back scattering on NFIRAOS performance are evaluated with the TMT MK13N site $25 \%$ and $50 \% C_{n}^{2}$ profiles, and telescope zenith angles at 0,30, 45, and 60 degrees. The constrained matched filter and the wavefront reconstructor are updated to account for the pixel background created by fratricide scattering.

The incremental wavefront is computed as the quadrature difference between each case and the baseline case where no fratricide effect is considered. For the baseline, the wavefront error in LGS controlled modes are shown in unit of $\mathrm{nm}$. For the other cases with fratricide effect included, the quadrature difference between the wavefront error in LGS controlled modes in that case and the baseline case is shown in unit of $\mathrm{nm}$. The percentage of ruined subapertures are also shown, which refer to those that have the measurement error (in milli-arcsecond) at least doubled due to the fratricide effect.

\subsection{Nominal 35" Radius LGS Asterism}

The results for our nominal LGS asterism (5 LGS on a 35" radius pentagon with an extra one on axis) are shown in Table 3. The following describes each case in the table in detail.

- Case "No fratricide": the baseline where no back scatter is considered.

- Case "not calibrated": the worst case where the fratricide scattering pattern changes so quickly that it can not be calibrated.

- Case "100\% calibrated": the best case where the fratricide scattering pattern is very stable so that it can be completely calibrated during the matched filter updating through LGS beam dithering. Only the photon noise is impacting the performance.

- Case " $80 \%$ calibrated": the most realistic case where the fratricide varies in short term but can be calibrated at $80 \%$ level.

\subsection{Various LGS Asterism Radius}

We also studied the dependence of the fratricide effect on LGS asterism diameter. With larger LGS asterism radius, more subapertures will be affected. But, contrary to what one might expect, the incremental wavefront error caused by the fratricide effect does not increase with larger LGS asterism diameter. Figure 3 shows the back scatter patterns of WFS 1 at zenith angle 60 for LGS asterism radius of 35" and 120". As we can see, although the number of affected subapertures becomes larger with larger LGS asterism radius, the intensity of the back scattered light in each subaperture becomes less. This is because as the angle between beam increases, the back scatter is integrated on a shorter path through the beam. The performance results are shown in Table 4. The impact of fratricide gradually decreases with increasing LGS asterism radius for zenith angle 60 degree and stays pretty stable for smaller zenith angles. $80 \%$ calibration of the back scatter is assumed. Here the fratricide effect are computed with various LGS asterism diameter, but the simulation and LGS turbulence tomography are carried out using nominal 35" radius LGS asterism to avoid changing multiple parameters at once. We believe this combination is enough to show the dependence of system performance with LGS asterism radius. 


\begin{tabular}{|c|c|c|c|c|c|c|c|c|c|}
\hline \multicolumn{1}{|c|}{$C_{n}^{2}$ Profile } & \multicolumn{4}{|c|}{$25 \% \mathrm{Mk} 13 \mathrm{~N}$} & \multicolumn{4}{c|}{$50 \% \mathrm{Mk} 13 \mathrm{~N}$} \\
\hline \multicolumn{2}{|c|}{ Zenith angle (deg) } & 0 & 30 & 45 & 60 & 0 & 30 & 45 & 60 \\
\hline \hline No fratricide & LGS WFE (nm) & 94.6 & 106.1 & 130.2 & 193.7 & 118.0 & 131.5 & 159.7 & 234.0 \\
\hline not calibrated & Incr. WFE (nm) & 9.8 & 15.5 & 29.2 & 84.3 & 11.8 & 19.7 & 38.9 & 116.8 \\
\hline not calibrated & Ruined subaps & $2.7 \%$ & $4.1 \%$ & $6.8 \%$ & $12.0 \%$ & $2.7 \%$ & $4.4 \%$ & $7.3 \%$ & $12.5 \%$ \\
\hline $80 \%$ calibrated & Incr. WFE (nm) & 3.6 & 4.0 & 7.8 & 20.5 & 1.4 & 4.7 & 10.3 & 30.6 \\
\hline $80 \%$ calibrated & Ruined subaps. & $0.4 \%$ & $0.7 \%$ & $1.4 \%$ & $4.3 \%$ & $0.4 \%$ & $0.7 \%$ & $1.5 \%$ & $4.6 \%$ \\
\hline $100 \%$ calibrated & Incr. WFE (nm) & 3.01 & 3.61 & 4.03 & 13.5 & 3.0 & 4.8 & 6.5 & 18.9 \\
\hline $100 \%$ calibrated & Ruined subaps & $0.2 \%$ & $0.4 \%$ & $0.7 \%$ & $1.8 \%$ & $0.2 \%$ & $0.4 \%$ & $0.7 \%$ & $1.8 \%$ \\
\hline
\end{tabular}

Table 3: Impact of fratricide on NFIRAOS performance with MK13N 25\% and $50 \% C_{n}^{2}$ profile for the nominal 35" radius LGS asterism. (Four turbulence seeds with 500 time steps each are used to do the averaging.)
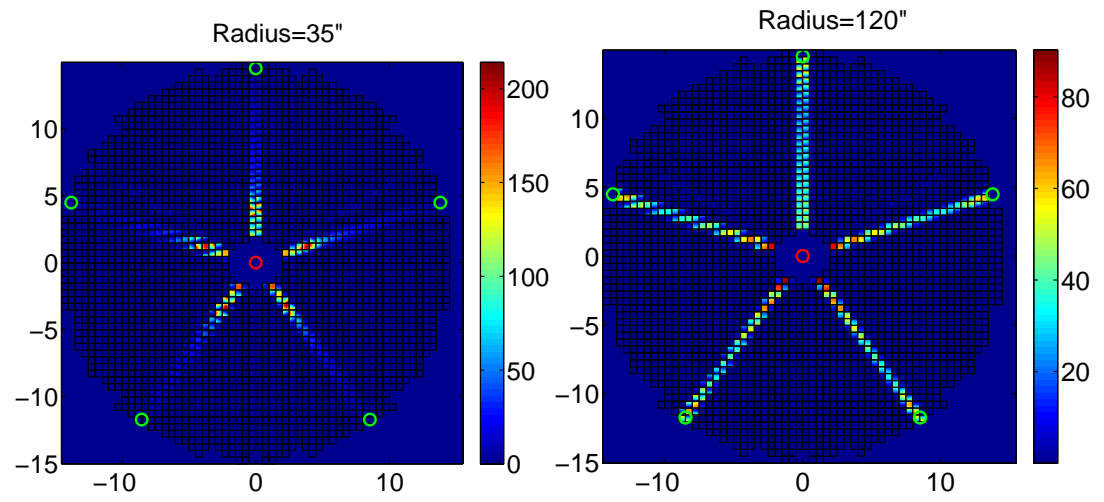

Figure 3: the back scatter patterns of WFS 1 at zenith angle 60 for LGS asterism radius of $35 "$ and $120 "$ ". Although the number of affected subapertures becomes larger with larger LGS asterism radius, the intensity of the back scattered light becomes less.

\begin{tabular}{|c|c|c|c|c|c|c|c|c|c|}
\hline \multicolumn{1}{|c|}{$C_{n}^{2}$ Profile } & \multicolumn{4}{|c|}{$25 \% \mathrm{Mk} 13 \mathrm{~N}$} & \multicolumn{4}{c|}{$50 \% \mathrm{Mk} 13 \mathrm{~N}$} \\
\hline \multicolumn{2}{|c|}{ Zenith angle (deg) } & 0 & 30 & 45 & 60 & 0 & 30 & 45 & 60 \\
\hline \hline $35 "$ & Incr. WFS (nm) & 3.6 & 4.0 & 7.8 & 20.5 & 1.4 & 4.7 & 10.3 & 30.6 \\
\hline $35 "$ & Ruined subaps & $0.4 \%$ & $0.7 \%$ & $1.4 \%$ & $4.2 \%$ & $0.4 \%$ & $0.7 \%$ & $1.5 \%$ & $4.6 \%$ \\
\hline $60 "$ & Incr. WFS (nm) & 2.9 & 4.3 & 8.8 & 19.6 & 1.0 & 6.5 & 11.2 & 27.1 \\
\hline $60 "$ & Ruined subaps & $0.7 \%$ & $0.9 \%$ & $1.8 \%$ & $4.5 \%$ & $0.6 \%$ & $0.9 \%$ & $1.9 \%$ & $5.0 \%$ \\
\hline $90 "$ & Incr. WFS (nm) & 4.6 & 6.1 & 8.6 & 18.3 & 2.0 & 6.3 & 12.9 & 24.2 \\
\hline $90 "$ & Ruined subaps & $0.4 \%$ & $0.7 \%$ & $1.3 \%$ & $3.3 \%$ & $0.4 \%$ & $0.7 \%$ & $1.4 \%$ & $3.8 \%$ \\
\hline $120 "$ & Incr. WFS (nm) & 5.5 & 4.9 & 8.3 & 16.2 & 3.7 & 8.2 & 10.3 & 20.1 \\
\hline $120 "$ & Ruined subaps & $0.3 \%$ & $0.6 \%$ & $0.9 \%$ & $1.1 \%$ & $0.3 \%$ & $0.5 \%$ & $1.0 \%$ & $1.4 \%$ \\
\hline $150 "$ & Incr. WFS (nm) & 5.1 & 5.9 & 8.5 & 12.0 & 5.0 & 7.4 & 11.3 & 16.9 \\
\hline $150 "$ & Ruined subaps & $0.2 \%$ & $0.4 \%$ & $0.8 \%$ & $0.1 \%$ & $0.2 \%$ & $0.4 \%$ & $0.8 \%$ & $0.1 \%$ \\
\hline
\end{tabular}

Table 4: The incremental wavefront error caused by the fratricide for various asterism radius. The impact of fratricide gradually decreases with increasing LGS asterism radius for zenith angle 60 degree and stays pretty stable for smaller zenith angles. 


\section{CONCLUSION}

We have presented the analysis on fratricide effects, caused mainly by Rayleigh back scattering, and show that the performance impact is less than $10 \mathrm{~nm}$ for zenith angles up to 45 degrees. The impact of the fratricide effect does not increase with larger LGS asterism radius. The lower sensitivity to noise of constrained matched filter used for pixel process is a major plus compared to other centroiding algorithms. The constrained matched filter put most of its weights on pixels where the reference image (the time averaged subaperture image) has large values and changes most as tip/tilt varies. The increased background due to Rayleigh back scattering on pixels where the weights are small does not appreciably affect the gradient measurements.

\section{Acknowledgment}

This work is supported by the Thirty Meter Telescope (TMT) project. The authors gratefully acknowledge the support of the TMT partner institutions. They are the Association of Canadian Universities for Research in Astronomy (ACURA), the California Institute of Technology and the University of California. This work was supported as well by the Gordon and Betty Moore Foundation, the Canada Foundation for Innovation, the Ontario Ministry of Research and Innovation, the National Research Council of Canada, the Natural Sciences and Engineering Research Council of Canada, the British Columbia Knowledge Development Fund, the Association of Universities for Research in Astronomy (AURA) and the U.S. National Science Foundation

\section{APPENDIX}

\subsection{Fourier transform of the LLT pupil amplitude function $L_{i}$}

The Fourier transform pupil amplitude function for a Gaussian beam

$$
L_{i}(\mathbf{x})=\exp \left[-\left(\mathbf{x}-\left(\mathbf{r}_{i}+h \boldsymbol{\theta}_{i}\right)\right) / 2 \sigma^{2}\right] .
$$

can be computed analytically.

$$
\hat{L}_{i}(\boldsymbol{\kappa})=2 \pi \sigma^{2} \exp \left[-2 \sigma^{2} \pi^{2}|\boldsymbol{\kappa}|^{2}-2 \pi i \boldsymbol{\kappa} \cdot\left(\mathbf{r}_{i}+h \boldsymbol{\theta}_{i}\right)\right] .
$$

But for the truncated beam, it has to be computed numerically.

\subsection{Fourier transform of the subaperture function $S_{0}$}

The subaperture mask function is a rectangular function that defines the pupil of a subaperture, i.e.,

$$
S_{0}(\mathbf{x})=\sqcap(x / d) \sqcap(y / d) .
$$

The Fourier transform is simply

$$
\hat{S}_{0}(\boldsymbol{\kappa})=d^{2} \operatorname{sinc}\left(k_{x} d\right) \sin \left(k_{y} d\right),
$$

where

$$
\operatorname{sinc}(x) \equiv \frac{\sin (\pi x)}{\pi x} .
$$

\subsection{Fourier transform of the detector pixel influence function}

We define the detector pixel influence function in a polar coordinate CCD in the local coordinate system with axes aligned along the radial and azimuthal directions. The pixel function can be modeled as a rectangular function that defines the edge of the pixel, convolved by a Gaussian blurring function that models the charge diffusion:

$$
d_{j k}^{\prime}\left(\phi_{r a}\right)=\sqcap\left(\phi_{r} / \Delta\right) \sqcap\left(\phi_{a} / \Delta\right) \star \frac{1}{2 \pi \phi_{b l u r}^{2}} \exp \left[-\left(\phi_{r}^{2}+\phi_{a}^{2}\right) /\left(2 \phi_{\text {blur }}^{2}\right)\right] .
$$

The Fourier transform of this model is

$$
\hat{d}^{\prime}\left(u_{r a}\right)=\Delta^{2} \operatorname{sinc}\left(u_{r} \Delta\right) \operatorname{sinc}\left(u_{a} \Delta\right) \exp \left[-2 \pi^{2} \phi_{b l u r}^{2}\left(u_{r}^{2}+u_{a}^{2}\right)\right] .
$$


The relation between the coordinates in the local radial-azimuthal coordinate system and the global Cartesian system is

$$
\phi_{r a}=R_{\alpha}^{T} \phi_{x y}
$$

where $R_{\alpha}$ is the transformation matrix for a rotation of angle $\alpha$ :

$$
R_{\alpha}=\left[\begin{array}{cc}
\cos \alpha & -\sin \alpha \\
\sin \alpha & \cos \alpha
\end{array}\right]
$$

Therefore

$$
d_{j k}\left(\phi_{x y}\right)=d_{j k}^{\prime}\left(R_{\alpha}^{T} \phi_{x y}\right)
$$

and its Fourier transform is

$$
\begin{aligned}
\hat{d}_{j k}\left(u_{x y}\right) & =\int \mathrm{d}\left(R \phi_{r a}\right) d_{j k}^{\prime}\left(\phi_{r a}\right) \exp \left[-2 \pi i\left(R \phi_{r a}\right)^{T} u_{x y}\right] \\
& =\left|R_{\alpha}^{-1}\right| \int \mathrm{d} \phi_{r a} d_{j k}^{\prime}\left(\phi_{r a}\right) \exp \left[-2 \pi i \phi_{r a}^{T}\left(R^{T} u_{x y}\right)\right] \\
& =\hat{d}_{j k}^{\prime}\left(R_{\alpha}^{T} u_{x y}\right) .
\end{aligned}
$$

Or more explicitly,

$$
\begin{aligned}
\hat{d}_{j k}\left(u_{x y}\right)= & \Delta^{2} \operatorname{sinc}\left[\left(u_{x} \cos \theta+u_{y} \sin \theta\right) \Delta\right] \operatorname{sinc}\left[\left(u_{x} \sin \theta-u_{y} \cos \theta\right) \Delta\right] \\
& \cdot \exp \left[-2 \pi^{2}\left(u_{x}^{2}+u_{y}^{2}\right) \phi_{b l u r}^{2}\right] .
\end{aligned}
$$

\section{REFERENCES}

[1] P. L. Wizinowich, et al., "The W. M. Keck Observatory Laser Guide Star Adaptive Optics System: Overview," Pub. Astron. Soc. Pacific 118, 297-309 (2006).

[2] M. Boccas, et al., "Laser guide star upgrade of Altair at Gemini North," in "SPIE", vol. 6272 (2006)

[3] M. Le Louarn, N. N. Hubin, and R. Arsenault, "Adaptive optics for second-generation VLT instruments," in "SPIE Conference Series", vol. 5490 (2004), pp. 248-259.

[4] B. Ellerbroek, et al., "Progress toward developing the TMT adaptive optical systems and their components," in "SPIE Conference Series,", vol. 7015 (2008).

[5] E. Diolaiti,et al."A preliminary overview of the multiconjugate adaptive optics module for the E-ELT," in "SPIE Conference Series,", vol. 7015 (2008)

[6] H. C. V. de Hulst, Light Scattering by Small Particles (Wiley, New York, 1957).

[7] E. J. McCartney, Optics of the atmosphere: Scattering by molecules and particles (1976).

[8] A. Bucholtz, "Rayleigh-scattering calculations for the terrestrial atmosphere," Appl. Opt. 34, 2765 (1995).

[9] S. S. Srivastava, N. K. Vyas, J. Rai, and B. Kartikeyan, "On the different approaches of Rayleigh optical depth determination," Advances in Space Research 44, 1058-1066 (2009).

[10] U.S., US Standard Atmosphere Supplement (U.S.Government Print Office, Washington, D.C., 1966).

[11] S. N. Seinfeld, John H. ; Pandis, Atmospheric Chemistry and Physics - From Air Pollution to Climate Change (John Wiley \& Sons, 2006).

[12] R. J. Sica, Z. A. Zylawy, and P. S. Argall, "Ozone corrections for rayleigh-scatter temperature determinations in the middle atmosphere," Journal of Atmospheric and Oceanic Technology 18-7, 1223 (2001).

[13] A. T. Young, http://mintaka.sdsu.edu/GF/explain/extinction/extintro.html (2008).

[14] Gemini, "http://sciopsedit.gemini.edu/sciops/telescopes-and-sites/observing-conditionconstraints/extinction," (2009).

[15] G. E. Shaw, "Aerosols at Mauna Loa: optical properties." Journal of Atmospheric Sciences 36, 862-869 (1979).

[16] T. I. G. Eguchi, Nawo Yokota, "Characteristics of cirrus clouds from icesat/glas observations," Geophysical Research Letters 34 (2007).

[17] L. Gilles and B. L. Ellerbroek, "Constrained matched filtering for extended dynamic range and improved noise rejection for Shack-Hartmann wavefront sensing," Optics Letters 33, 1159-+ (2008). 